\title{
Global review of assisted diagnosis tools using medical database and artificial intelligence methods to improve complex disease diagnosis
}

Anne Blériot ( $\square$ anne.bleriot@madisphileo.com )

Madis Phileo

Franck Le Meur

TechToMed

Guillaume De Chamisso

Madis Phileo

\section{Research Article}

Keywords: Symptom checkers, artificial intelligence, medical database, rare disease, diagnosis

Posted Date: January 29th, 2021

DOI: https://doi.org/10.21203/rs.3.rs-157785/v1

License: (c) (i) This work is licensed under a Creative Commons Attribution 4.0 International License.

Read Full License 


\title{
Global review of assisted diagnosis tools using medical database and artificial intelligence methods to improve complex disease diagnosis
}

Franck Le Meur, PhD, CEO, TechToMed

Anne Blériot, PhD, scientific consultant, Madis Phileo

Guillaume De Chamisso, consultant, Madis Phileo

\section{Keywords}

Symptom checkers, artificial intelligence, medical database, rare disease, diagnosis

\begin{abstract}
Millions of people worldwide suffer from a rare disease. Many among them without a definitive diagnosis. The object of this study was to identify, evaluate and rate the most appropriate and promising technological tools to help patients effectively navigate their rare disease journey and reduce their exposure to diagnostic wandering.

For the analysis of available tools, products were separated into four technology categories: artificial intelligence, assisted anamnesis (symptom checkers), awareness/patient selfscreening, direct identification of patients via screening. Tools were then ranked according to two criteria: impact on patients and operability and subsequently narrowed down further for more in-depth analysis. In two separate advisory board meetings, the most promising tools were then evaluated by healthcare professionals and patient representatives, respectively.

Across four categories and 107 different tools and means for the purposes of reducing diagnostic wandering in rare disease patients, instruments such as Symptoma, Isabel or FindZebra emerged as the favored solution on both advisory board meeting groups. Symptoma was selected to be further evaluated in a comprehensive pilot study with cardiologists and general practitioners in a real-world setting.
\end{abstract}

\section{Introduction}

Rare diseases are traditionally defined as those diseases that affect only a small part of the population. However, there is no single, internationally harmonized definition for rare diseases and therefore the term "rare disease" as a concept is interpreted through different quantitative terms. While in the USA less than 200,000 persons affected [1], or $<0.06 \%$, may be affected by a so-called rare disease, the EU's definition is that if less than 5 per 10,000 inhabitants [2] (equivalent to $0.05 \%$ ) suffer from a particular pathology, it is considered rare. Around $85 \%$ of diseases fall within this definition. Some even demonstrate a point prevalence of $<1$ per $1,000,000$, making these ailments even rarer. [3]

However, when rare diseases are considered in their totality, between 6,000 and 8,000 diseases are covered by this definition and approximately $6-8 \%$ of the population is affected by at least one of these pathologies [4]. 
While currently millions of people worldwide suffer from a rare disease, many of them do not have a definitive diagnosis. This inconsistency is driven by two main factors:

1. The patient has not yet been accurately diagnosed because the symptoms have not yet been correctly classified, categorized or assessed;

2. The patient suffers from a disease for which there is no diagnostic test available as of yet, because neither the disease nor its cause have been amply specified or defined. This category of diseases is referred to as "Syndrome without a name" (SWAN). [5]

Even for those patients who do not suffer from SWAN and were a disease has already been properly identified and described, the path to a definitive and correct diagnosis may be lengthy and arduous. While it is difficult to determine the actual time from symptom onset to diagnosis, case studies and patient reports clearly show that it can take several years, sometimes even decades, for patients with rare diseases to be properly diagnosed. [6]

A late or missing diagnosis may have serious or even life-threatening consequences for the patient. Furthermore, the lack of a proper diagnosis affects the patient's every day and social life and his or her personal and professional environment due to a lack of knowledge about the actual disease. In addition to the patient and his or her family, society in general and governmental health resources are affected and possibly disproportionately consumed. [7]

There are several drivers that disallow an accurate rare disease diagnosis or that allow for an initial misdiagnosis. Primarily, the lack of specific knowledge of the treating physician about the plethora of possible rare diseases or the application of unsuitable diagnostic tests to arrive at the appropriate clinical conclusion, are the main factors. [5]

Over the past decades, the development of innovative technologies for presenting, aggregating and analyzing data and health information has continued to advance. This 
progress has especially proliferated in the field of medicine and the evaluation of symptoms, diseases and their associated differential diagnoses. Due to the steadily increasing amount of available knowledge and data, there is a significant potential for new technologies when it comes to patient screening, diagnostic guidance or predictive analysis.

There are already numerous such tools available on the market. Some manufacturers claim to be able to satisfy this unmet need. Others may not directly offer a defined intended diagnostic use, but the product's functionality may be utilized to support the diagnosis of rare diseases in the future. The object of this study was to identify and evaluate the most appropriate and promising tools to help patients effectively navigate their rare disease journey.

\section{Methods}

For the analysis of available tools that may be used to diagnose patients with rare diseases, all identified available products were separated into four technology categories:

- Artificial intelligence

- Assisted anamnesis (symptom checkers)

- Awareness/Patient self-screening

- $\quad$ Direct identification of patients via screening

In order to complete a reproduceable and comprehensive picture of available tools worldwide, all obtainable products were identified through searches of privileged, internal company data (Madis Phileo, France, with the financial support of Pfizer France for this project), search engines (e.g.: Google), social media (e.g.: Twitter), the UNIR White Paper [8] and the scientific literature indexing instrument, PubMed. Only tools that covered a comprehensive field of indications were considered - disease-specific tools were excluded in a subsequent step. 
After a comprehensive search and the generation of results, an inclusive list of products was assembled. Subsequently, a three-step-approach was followed to rank and assess the resulting tools.

During the „pre-selection process“, all tools were evaluated according to two criteria:

- Impact on patients: usefulness and perceived effectiveness in reducing diagnostic wandering (expert assessment by representatives of the company Madis Phileo, France)

- Operability: ease of use and/or implementation of the tool for a physician or patient (existing experience or a use case with the specific tool and/or economic model)

The evaluation was carried out on a 6 -level scale, with 0 being the lowest possible and 5 the best possible rating in each category. In a first step, impact and operability scores were averaged and then the top 38 tools were selected to allow for a representative sampling of the highest ranked tools in each of the four categories.

In a second step, the top-ranked tools of each category were narrowed down to a total of 23 by experts from Madis Phileo based on available and representative content which was considered critical as input for discussions and evaluations during Advisory Board Meetings (ABM). Tools that lacked usable content and an opportunity for substantive interaction and evaluation, which was a requirement for $A B M$ review, were excluded. The top 2 to 3 tools from each category were then selected according to qualitative criteria of highest rank within a category and a capability for live testing. This final selection of 12 tools was submitted to the ABM for evaluation. These ABMs were conducted with two sets of participants: once with a selection of five health care professionals (two endocrinologists, one general practitioner, one cardiologist, one pharmacist) and secondly with patient delegates from five different 
pathologies (Sickle cell anemia, Lymphangioleiomyomatosis, Amyloidosis, Acromegaly, and growth pathologies).

As a primer, the ABM participants were initially asked about their experiences and opinions about the reasons for diagnostic wandering in patients with rare diseases. Additionally, the question was posed to participants on where they see challenges in daily practice and how this situation may be addressed and improved.

Once the pre-selected tools were presented to the ABMs, they were asked again for their opinions and to state which concepts seem the most feasible and promising to address the aforementioned needs.

\section{Results}

Comprehensive information about relevant tools that may support optimized diagnosis from the sources mentioned above were categorized and their relevance examined. A total of 107 tools were identified, which were considered in the following pre-selection process. During pre-selection, all tools were rated and 38 out of 107 tools $(35.5 \%)$ received a score of 5 or higher, when averaging their impact- and operability scores. The specifics are summarized in Table 1.

\begin{tabular}{|l|l|l|l|r|}
\hline Tool name & Manufacturer & Category & $\begin{array}{l}\text { Score: } \\
\text { Impact }\end{array}$ & $\begin{array}{l}\text { Score: } \\
\text { Operability }\end{array}$ \\
\hline AideDiag & Dr Bernard Chassaing & AA & 4 & 4 \\
\hline Alexa Diagnostics & Amazon.com Inc. & ID & 1 & 5 \\
\hline Anamnese.me & $\begin{array}{l}\text { Jérôme Bourreau, } \\
\text { Président }\end{array}$ & AA & 3 & 5 \\
\hline Assistant Médical & Dr Jean Thouluc, & AA & 4 & 5 \\
\hline
\end{tabular}




\begin{tabular}{|c|c|c|c|c|}
\hline $\begin{array}{l}\text { Automatic detection of } \\
\text { Acromegaly }\end{array}$ & $\begin{array}{l}\text { Peking Union Medical } \\
\text { College Hospital } \\
\text { Main author: Xiangyi Kong }\end{array}$ & ID & 4 & 3 \\
\hline Babylon Health & Babylon & TC & 4 & 5 \\
\hline $\begin{array}{l}\text { BP neural network } \\
\text { suicide } \\
\text { prediction mode }\end{array}$ & $\begin{array}{l}\text { Lyu J, Department of } \\
\text { Public Health, Weifang } \\
\text { Medical University, China }\end{array}$ & ID & 1 & 4 \\
\hline $\begin{array}{l}\text { Computer vision } \\
\text { algorithm }\end{array}$ & $\begin{array}{l}\text { Oxford University, Quentin } \\
\text { Ferry }\end{array}$ & ID & 4 & 2 \\
\hline $\begin{array}{l}\text { Computer Vision for } \\
\text { Assistive Medical } \\
\text { Diagnosis From Faces }\end{array}$ & $\begin{array}{l}\text { University of Oulu, Finland } \\
\text { Author: Jérôme Thevenot }\end{array}$ & ID & 4 & 2 \\
\hline CrowdMed & CrowdMed & $A D$ & 4 & 4 \\
\hline DeepGestalt & & ID & 5 & 2 \\
\hline DocForYou & $\begin{array}{l}\text { Jean-Marie Catsellucci, } \\
\text { CEO }\end{array}$ & $A D$ & 4 & 2 \\
\hline DocResponse & DocResponse & $\mathrm{AA}$ & 4 & 5 \\
\hline Dr. Warehouse & Société française Codoc & IA & 4 & 3 \\
\hline eVillage platform & Cello Health & IA & 2 & 4 \\
\hline Face2Gene & FDNA & ID & 5 & 2 \\
\hline $\begin{array}{l}\text { Facebook - Suicide } \\
\text { prediction }\end{array}$ & Facebook & ID & 3 & 2 \\
\hline FindZebra & FindZebra & AA & 3 & 2 \\
\hline Google & Google LLC & $A D$ & 4 & 2 \\
\hline Health29 & Foundation29 & $\mathrm{IA}$ & 4 & 3 \\
\hline Isabel Healthcare & Isabel Healthcare Inc. & $\mathrm{AA}$ & 5 & 5 \\
\hline LIVI & LIVI & $\mathrm{TC}$ & 2 & 4 \\
\hline
\end{tabular}




\begin{tabular}{|c|c|c|c|c|}
\hline $\begin{array}{l}\text { Microsoft facial } \\
\text { recognition }\end{array}$ & Microsoft & ID & 4 & 2 \\
\hline MédecinDirect & & $\mathrm{TC}$ & 3 & 5 \\
\hline Moon & Diploid & $\mathrm{IA}$ & 5 & 4 \\
\hline PedAM & & IA & 3 & 2 \\
\hline Phenomizer & Phenomics & AA & 3 & 2 \\
\hline Physician Cognition & & $\mathrm{AA} / \mathrm{AD}$ & 4 & 4 \\
\hline PreMedicus & & $A D$ & 4 & 4 \\
\hline $\begin{array}{l}\text { Rare Disease Discovery } \\
\text { prediction engine }\end{array}$ & University of Lleida & $\mathrm{IA}$ & 4 & 3 \\
\hline SAGIT & Andrea Giustina & AA & 4 & 2 \\
\hline SimulConsult & SimulConsult & AA & 2 & 4 \\
\hline SOCRATE & Sanofi Genzyme & $\mathrm{AA}$ & 2 & 4 \\
\hline Sympto Check & $\begin{array}{l}\text { Docteur Clic (Dr Loïc } \\
\text { Etienne) }\end{array}$ & $A D$ & 3 & 4 \\
\hline Symptoma & Symptoma GmbH & AA & 5 & 4 \\
\hline Treatable-id.org & Clara D M van Karnebeek & AA & 4 & 4 \\
\hline Twitter data analysis & $\begin{array}{l}\text { The Zucker Hillside } \\
\text { Hospital, NY (USA) } \\
\text { Author: Michael L. } \\
\text { Birnbaum }\end{array}$ & ID & 1 & 4 \\
\hline UDN Gateway & $\begin{array}{l}\text { The National Human } \\
\text { Genome Research } \\
\text { Institute's } \\
\text { Undiagnosed Diseases } \\
\text { Network (UDN) }\end{array}$ & $A D$ & 2 & 4 \\
\hline
\end{tabular}


Out of the 38 highest scoring tools, the top-ranked instruments of each category were further narrowed to 23 and subsequently 12 tools were entered into the ABM review for assessment based on the aforementioned methodology. This final set served as the evaluation input for the ABMs.

The advisory board with five patient representatives was held in May 2019. After reviewing and discussing the available selection of technologies and tools, "assisted anamnesis for doctors" (in comparison with symptom checkers dedicated to patients) was named as the preferred technology category by the patient ABM. Additionally, a module for 'a patient in the waiting room preparing for a clinical examination and interview' was named as a promising opportunity to improve patient-physician-communication. As a suggestion for further improvement, a list of reference centers for more detailed testing and evaluation should be provided by the tool in case of a suspected rare disease.

In order to further educate patients and provide more in-depth information about the topic of rare diseases, the Google search bar is a critical tool to highlight disease awareness websites. This initially requires accurate 'real-life patient wording' (i.e.: Medical Words, Medwords) about a particular disease and then an effective algorithm to reference the right sources and index appropriate websites.

The professional health care providers ABM consisting of five participants was held in June 2019.

During the course of the meeting, important aspects for improved and accelerated diagnosis of rare diseases were discussed. The general factors identified as contributing to better diagnosis of rare diseases were increased attention by medical professionals to potential differential diagnoses and better perception and uptake of patient communication and information. As for the patient delegate ABM, assisted anamnesis was named as the most promising technology category to support diagnosis of rare diseases by the medical 
professionals. Technology-supported preparation efforts for the doctor's consultation was also mentioned as an important tool for improving the doctor-patient consultation. However, in order to not cause patient anxiety, preliminary results of the tool's algorithm should initially be reserved for the physician.

The members of the ABM had a positive attitude towards the introduction of new technologies and expect great benefits from innovation-driven, automated improvements to diagnostic efforts. Symptoma ${ }^{\circledR}$, an interactive, chatbot-based, class I medical device software, was specifically identified as a superior and promising tool to make diagnosis of rare diseases more accurate and reduce diagnostic wandering.

\section{Discussion}

Unacceptably long periods for time-to-diagnosis for many rare disease patients are indicative of clear deficits in this segment of health care provision. Efforts have been made in the past to reduce or even eliminate these deficits by optimizing existing processes in health care systems, but so far with only moderate success. New technologies for the utilization and exploitation of big data and artificial intelligence are promising alternatives to address this unmet health care need. Especially the intelligent processing of large amounts of health data, medical information and knowledge by appropriate tools will support the efforts in identifying rare disease patients sooner and more accurately. [9] This expectation is also supported by governmental institutions such as the German National Action League for People with Rare Diseases (NAMSE) or the UK Department of Health, which anticipate to establish software-supported diagnostic systems in their national health care action plans. [10], [11]

Over the course of this evaluation, interest in and the significance of these technologies was confirmed by representatives of each ABM - the HCPs and patient delegates. In particular, the use of tools supporting anamnesis efforts seems promising to all ABM participants. While 
the use of the internet to search for health care answers and symptom-related information is proliferative and widely popular amongst both patients and professionals [12], there is a need to systematically process both the information on symptoms that is provided by patients as well as the more specific information on potential diagnoses that is then presented to the HCP. Tools for assisted anamnesis which support diagnosis must, on the one hand, be able to amplify relevant signals from the patient and display possible clinical pictures, but also be capable of strengthening the digital link between doctor and patient in order to efficiently transport and process information between the two parties. Over the course of this evaluation, Symptoma's chatbot symptom checker was identified as a superior product that meets both of these requirements and was deemed to be particularly interesting to the HCP ABM participants. Based on these conclusions, a handful of symptom checkers should be considered as powerful tools in answering the critical challenge of diagnostic wandering (e.g.: Symptoma, Isabel, FindZebra).

Symptoma allows the user to obtain a list of possible medical causes ranked by likelihood of accuracy. In a recent study authored by Martin et al. the accuracy of the Symptoma chatbot in identifying COVID-19 demonstrated that Symptoma can accurately distinguish COVID-19 cases in $96.32 \%$ of clinical vignettes. [13] Similarly, in an updated evaluation from the Semigran et al study [14], the diagnostic accuracy of symptom checkers from an ear, nose and throat perspective was evaluated recently. With respect to the ENT cases, Symptoma's symptom checker demonstrated superior diagnostic accuracy of $64.3 \%$ (at top 1 ), $92.9 \%$ (at top 3 ) and $100 \%$ (at top 10$)$ in comparison to Isabel's $(21,4 \% ; 40,4 \% ; 61,9 \%)$ and FindZebra's (26,2\%; 42,9\%; 54,8\%) symptom checkers. [15]

These listings are the result based on the symptoms the user entered and the subsequent interactive queries guided by an intelligent chatbot. This is in line and consistent with the requests that were made by both of the ABM participants: patient delegates and HCP representatives. 


\section{Conclusion}

Across four categories of 107 different tools and means for the purposes of reducing diagnostic wandering in rare disease patients, instruments such as Symptoma, Isabel or FindZebra emerged as the favored solution among patient delegates and HCPs. Symptoma was selected to be evaluated in a comprehensive pilot study with cardiologists and general practitioners in a real-world setting testing the tool's acceptance and usage.

\section{References:}

[1] O. of the Commissioner, "Rare Diseases at FDA," FDA, Feb. 20, 2020. https://www.fda.gov/patients/rare-diseases-fda (accessed Jul. 12, 2020).

[2] Anonymous, "Rare diseases," Gesundheitswesen - European Commission, Nov. 25, 2016. https://ec.europa.eu/health/non_communicable_diseases/rare_diseases_de (accessed Jul. 12, 2020).

[3] S. Nguengang Wakap et al., "Estimating cumulative point prevalence of rare diseases: analysis of the Orphanet database," Eur. J. Hum. Genet., vol. 28, no. 2, Art. no. 2, Feb. 2020, doi: 10.1038/s41431-019-0508-0.

[4] H. J. S. Dawkins et al., "Progress in Rare Diseases Research 2010-2016: An IRDiRC Perspective," Clin. Transl. Sci., vol. 11, no. 1, pp. 11-20, 2018, doi: 10.1111/cts.12501.

[5] S. Gainotti et al., "Meeting Patients' Right to the Correct Diagnosis: Ongoing International Initiatives on Undiagnosed Rare Diseases and Ethical and Social Issues," Int. J. Environ. Res. Public. Health, vol. 15, no. 10, 21 2018, doi: 10.3390/ijerph15102072.

[6] "SURVEY OF THE DELAY IN DIAGNOSIS FOR 8 RARE DISEASES IN EUROPE ('EURORDISCARE 2')," p. 2.

[7] EURORDIS, "INTERNATIONAL JOINT RECOMMENDATIONS TO ADDRESS SPECIFIC NEEDS OF UNDIAGNOSED RARE DISEASE PATIENTS," Oct. 2016.

[8] Sanofi, "UNIR White Paper."

[9] S. Ronicke, M. C. Hirsch, E. Türk, K. Larionov, D. Tientcheu, and A. D. Wagner, "Can a decision support system accelerate rare disease diagnosis? Evaluating the potential impact of Ada DX in a retrospective study," Orphanet J. Rare Dis., vol. 14, Mar. 2019, doi: 10.1186/s13023-019-1040-6.

[10] NAMSE, "Nationaler Aktionsplan für Menschen mit Seltenen Erkrankungen," 2013.

[11] UK Department of Health, "The UK Strategy for Rare Diseases," 2013.

[12] "Health Online 2013."

[13] A. Martin, et al., "An artificial intelligence-based first-line defence against COVID-19: digitally screening citizens for risks via a chatbot," bioRxiv., doi: 10.1101/2020.03.25.008805, 2020. 
[14] H. Semigran et al., "Evaluation of symptom checkers for self-diagnosis and triage: audit study," BMJ, 1756-1833, 10.1136/bmj.h3480, 2015.

[15] J. Nateqi et al., "From symptom to diagnosis-symptom checkers re-evaluated: Are symptom checkers finally sufficient and accurate to use? An update from the ENT perspective," HNO, 334-342, doi: 10.1007/s00106-019-0666-y, 2019.

\section{Declarations:}

- Ethics approval and consent to participate

This project is exempt from IRB review as no personal or health-related data were processed or accessed during the evaluation and no human subjects, cells or animals were subject to investigation.

- Consent for publication

All authors of the manuscript have read and agreed to its content and are accountable for all aspects of the accuracy and integrity of the manuscript in accordance with ICMJE criteria. The manuscript is original, has not already been published, and is not currently under consideration by another journal.

- Availability of data and material

The authors confirm that the data supporting the findings of this study are available within the article and its supplementary materials.

- Competing interests

There are no competing interest to declare.

- Funding

Pfizer supported Madis Phileo in this project with an unrestricted grant. Symptoma and the European Commission (grant agreement number 830017) supported writing efforts and publication fees. The design, execution, data analysis, and conclusions of the study were under the sole control and responsibility of Madis Phileo.

- Authors' contributions

Franck Le Meur, Anne Blériot and Guillaume De Chamisso contributed to the design and implementation of the study, to the analysis of the results and to the writing of the manuscript.

- Acknowledgements

We express our gratitude to Martin Popovic from CATALYST SCIENTIFIC for his independent medical writing support. We thank Pfizer, Symptoma and the European Commission for providing funding to support this study. 